Rev. Fac. Ing. - Univ. Tarapacá, vol. 14 Nº 1, 2006, pp. 95-101

\title{
AN OPTIMISATION-BASED APPROACH FOR DESIGNING INDIRECT SENSORS APPLIED TO AN INDUCTION MOTOR
}

\section{MÉTODO DE ESTIMACIÓN DE ESTADOS BASADO EN OPTIMIZACIÓN APLICADO A UN MOTOR DE INDUCCIÓN}

\author{
Gonzalo Valdés-González ${ }^{1}$ Héctor Valdés-González ${ }^{2} \quad$ Juan Zolezzi $^{3}$ \\ Recibido el 28 de junio de 2005, aceptado el 24 de enero de 2006 \\ Received: June 28, 2005 Accepted: January 24, 2006
}

\begin{abstract}
RESUMEN
Este artículo propone una novedosa estrategia aplicada al problema de estimación no lineal de estados en un motor de inducción (MI). Este método permite, en general, la estimación de variables de difícil acceso o que simplemente no se pueden medir. Lo cual es posible a través de mediciones indirectas, considerando un modelo dinámico del proceso y un algoritmo de estimación basado en optimización no lineal. El principal atractivo de esta estrategia de estimación de estados denominada MHSE (Moving Horizon State Estimation) en un MI, que permite conocer la magnitud del flujo, la velocidad o posición del rotor, es su simplicidad de implementación, sus buenas características de convergencia, su independencia de estructuras preestablecidas de modelos y su fácil sintonía. Resultados en simulación muestran la efectividad del método propuesto efectuando la estimación de la velocidad de un MI bajo diferentes puntos de operación.
\end{abstract}

Palabras clave: MHSE, estimación de estados, motor de inducción, optimización no lineal, horizonte móvil.

\begin{abstract}
This article proposes an innovative strategy to the problem of non-linear estimation of states in an induction motor, (IM). This method allows the estimation of variables that are difficult to access or that are simply impossible to measure. The estimation is made possible by using indirect measures, through the consideration of a dynamic model of the process and an estimation algorithm based on non-linear optimization. This state estimation strategy (a.k.a. Moving Horizon State Estimation or MHSE) in an IM allows the determination of the flux magnitude and the velocity or position of the rotor. Its principal advantages are the simplicity of its implementation, good convergence characteristics, independence from preestablished model structures, and easy tuning. Simulated results corroborate the effectiveness of the proposed method through estimates of the velocity of an IM under different operational situations.
\end{abstract}

Keywords: MHSE, state estimation, induction motor, non-linear optimization, moving horizon.

\section{INTRODUCTION}

Different industrial applications of IMs require good dynamic response and precise control of torque and/or velocity (in electric traction, machine tools, etc.) At present these requirements can be met through electronic control of the IM [1]. Whichever of the various control techniques is employed, the flux magnitude and the velocity or position of the rotor must be known with sufficient accuracy. Even in an application without many mechanical performance requirements, it may be necessary to know the flux and/or velocity to control an IM, especially when it is desirable to minimize losses. For the operation of closed loop IMs, conventional approaches employ a velocity sensor, which is usually mechanically linked to the axis of the motor. However, the use of these sensors has a number of inconveniences, among which are:

\footnotetext{
1 Escuela de Negocios, Universidad de Tarapacá, Av. 11 de Septiembre N²222, Casilla 6-D, Arica, Chile, gvaldes@uta.cl

2 Departamento de Computación e Informática, Universidad de Tarapacá, Av. 11 de Septiembre No 2222, Casilla 6-D, Arica, Chile, hvaldes@uta.cl

3 Departamento de Ingeniería Eléctrica, Universidad de Santiago, Av. Ecuador 3519, Casilla 10233, Santiago, Chile, jzolezzi@lauca.usach.cl
} 
- Higher operating costs

- Less dependability and robustness

- Less immunity to noise

- Greater maintenance

Due to these inconveniences, different methods have been proposed for estimating the velocity or position of the rotor in an IM that eliminate the need for sensors. There are different velocity-estimating strategies based on measures of tensions and currents in the stator, among which are extended observers such as EKF (Extended Kalman Filter) [2], and those of a non-linear nature such as UKF (Unscented Kalman Filter) [3]. Although these methods have given rise to a variety of applications, they have disadvantages such as the associated mathematical complexities, their limitation to certain types of processes (for example a hybrid process), non-guaranteed convergence, and the fact that they can only be implemented by specialists.

This paper presents a method that is designed to avoid these weaknesses, i.e. a method that guarantees convergence (by using non-linear models to carry out an estimation of states-given that the estimation strategy proposed allows the use of these models regardless of their structure), and that can be used by non-specialists [9], [12] as there are fewer parameters to configure. In order to achieve these objectives this article presents the development of an estimation algorithm for flux and velocity based on non-linear optimization of states, and validates it by means of simulations. This algorithm is called Moving Horizon State Estimation or MHSE.

The work is organized as follows: Initially, the non-linear estimation method MHSE is presented. This is followed by a presentation of the non-linear model of the induction motor which will be used for carrying out estimates. The non-linear estimation algorithm under consideration is then implemented, and applied to an IM through simulations that verify the benefits of the methods and the estimation strategy outlined previously.

\section{MOVING HORIZON STATE ESTIMATION}

\section{The problem of estimation}

Consider the generic non-linear model (1) for a typical process, where represents the system states vector, the input vector and the output or measurement vector.

$$
\begin{aligned}
& \frac{d x}{d t}=f(x, u) \\
& y=g(x)
\end{aligned}
$$

The first stage of the model (1) represents the dynamic evolution of the process. The second stage of the model represents the relationship between the inputs (not necessarily measurable), and the output measurements. It is assumed that the output is measured in certain discrete instances denoted $t_{0}, \ldots t_{k}$.

The objective of the estimation is to provide a state value $\hat{x}(t)$ where $t \leq t_{k}$, using the process model and the previous values of the input $\Lambda_{u}(t)=\left(u\left(t_{0}\right), \ldots, u\left(t_{k}\right)\right)$, and the output $\Lambda_{y}(t)=\left(y\left(t_{0}\right), \ldots, y\left(t_{k}\right)\right)$. An algorithm which considers the measurement $y\left(t_{k}\right)$ can be considered as a virtual sensor which can indirectly measure $x(t)$. The key component of such an algorithm is the function $\left(\Lambda_{u}(k), \Lambda_{y}(k)\right) \rightarrow \hat{X}=\left\{\hat{x}\left(t_{k}\right)\right\}$, which can be understood as a set of estimated states that are a type of inversion of the dynamic system associated to the system's past history. This set will only have one element if, and only if, the system is observable [4], which can be difficult to ascertain when considering a generalized non-linear system. It is therefore assumed that the model represented by (1) is perfectly known and observable.

In this case, the accuracy of the state vector $\hat{x}(t)$ depends on the level of noise in the measurement. A good estimator should be able to eliminate the effect of such noise. On the contrary, if there are perturbations in the system's states, in other words unexpected variations in one or more state variables, the estimating algorithm's role (in the present case the MHSE method) will be to follow these perturbations as quickly as possible, because this implies a modification of the state of the process.

With respect to estimation algorithms, there are various approximations proposed in the literature. The most famous non-linear model is EKF [2]-[5], which is an extension of the linear Kalman filter for a non-linear case with a linearized model. This implies that when EKF is applied to a non-linear system, it is not possible to guarantee the convergence and stability of the filter. 
Also problematic is the fact that EKF requires statistical knowledge (covariance matrices) regarding the impact of noise on the states and on the output, which are not readily obtainable. A bad approximation of the covariance matrices will lead to poor results in the estimation. By applying this technique non-linear estimations have been implemented for an IM [3], taking into consideration different classes of non-linear models [6], [7].

It has been possible to develop specific non-linear observers for certain classes of systems. In these instances, the convergence of the observers can be established although mathematical developments are required that are often beyond the expertise of the persons implementing them, and moreover, they are limited to the category of systems for which they were conceived [11], [12].

Taking into consideration the reasons presented above and the typical disadvantages of classical non-linear estimation strategies, this paper presents an algorithm based on non-linear optimization on a horizon which is finite and moving in time. This method would seem to be a better solution for industrial purposes in the estimation of states applied to an IM.

\section{The Principle behind the MHSE method}

The MHSE method involves replacing a dynamic estimation problem with a static non-linear optimization problem. The defining criterion for this type of problem is the sum of the quadratic error between the input and output measurements estimated by the process model during a predefined time horizon. The minimization of this criterion seeks to determine the estimated state $\hat{x}(t)$ at the beginning of the time horizon, such that this leads to a minimization between observed measurements and the predicted trajectory in the time horizon. It should be noted that in the linear case, the problem can be easily resolved and a formal solution can be established. Nonetheless, a non-linear analytic solution cannot be established for the problem, and it must be solved numerically through non-linear optimization which forms the basis of the proposed method.

First, we shall describe the optimization criterion of MHSE which must be minimized. We will consider the non-linear model described in (1), with a single output in order to simplify notation where $Y_{k}=\left[\begin{array}{llll}y_{s h} & y_{s h+1} & \ldots & y_{k}\end{array}\right]^{T}$ are the measurements of the output in the horizon under consideration, with $y_{i}$ equal to $y\left(t_{i}\right)$. The subscripts $s h$ and $k$ represent the origin of the horizon and present time respectively (end of the horizon). $\hat{x}_{s h}$ denotes the state vector at the beginning of the time horizon, such that we can predict the trajectory of $Y_{k}$ over the pre-selected time horizon on the basis of this vector.

$$
\left[\begin{array}{llll}
\hat{y}_{s h} & \hat{y}_{s h+1} & \cdots & \hat{y}_{k}
\end{array}\right]^{T}=G\left(\hat{x}_{s h}, t_{s h}, t_{k}\right)
$$

Where $G\left(\hat{x}_{s h}, t_{s h}, t_{k}\right)$ is defined as:

$$
G\left(x, t_{i}, t_{j}\right)=\left[\begin{array}{c}
g\left(x\left(t_{i}\right)\right) \\
g\left(F\left(x, t_{i}, t_{i+1}\right)\right) \\
\vdots \\
g\left(F\left(x, t_{i}, t_{j}\right)\right)
\end{array}\right]
$$

Likewise, $F\left(x, t_{i}, t_{l}\right)$ is expressed by:

$$
F\left(x, t_{i}, t_{l}\right)=x\left(t_{i}\right)+\int_{t_{i}}^{t_{l}} f(x(\tau), u(\tau)) d \tau
$$

The principle of the method then is the determination of the state vector $\hat{x}_{s h}$ such that:

$$
\hat{x}_{s h}^{*}=\arg \min \left[J\left(\hat{x}_{s h}\right)\right]
$$

From (5) criterion $\pi($.) can be rewritten as:

$$
J\left(\hat{x}_{s h}\right)=\frac{1}{2} R_{k}^{T} R_{k}
$$

Where $R_{k}$ is the residues vector, expressed as:

$$
R_{k}=Y_{k}-G\left(\hat{x}_{s h}, t_{s h}, t_{k}\right)
$$

The observability of the system (1) is restricted by the existence and uniqueness of the solution of problem (5), i.e. this solution will have a unique element if, and only if, the system is observable. For further details, see [4], [9], [12], [14].

MHSE algorithm based on the BFGS method

The purpose of the MHSE method is to calculate the state vector $\hat{x}_{k}$ in real time, based on $\hat{x}_{s h}^{*}$ obtained from the 
solution of (5). This algorithm can be described as having two principal parts. The first is a minimization stage and the second is a predictive stage, ideally described as follows:

1) Initialization of the states vector $\hat{x}_{s h}$

2) Search for the optimum states vector $\hat{x}_{s h}^{*}$

a) Prediction of measurements over the complete horizon: $G\left(\hat{x}_{s h}, t_{s h}, t_{k}\right)$

b) Calculation of $\nabla J\left(\hat{x}_{s h}\right)$

c) While $\nabla J\left(\hat{x}_{s h}^{i}\right) \neq 0$, do:

$$
\hat{x}_{s h}^{i+1}=\hat{x}_{s h}-M^{i} \nabla J\left(\hat{x}_{s h}^{i}\right)
$$

d) If $\nabla J\left(\hat{x}_{s h}^{i}\right)=0 \Rightarrow \hat{x}_{s h}^{*}=\hat{x}_{s h}^{i}$

3) Calculation of the states vector in present time:

$$
\hat{x}_{k}^{*}=F\left(\hat{x}_{s h}^{*}, t_{s h}, t_{k}\right)
$$

4) Return to step 2) to calculate $\hat{x}_{k+1}^{*}$, with

$$
\begin{aligned}
& s h \leftarrow s h+1 \\
& \hat{x}_{s h}=F\left(\hat{x}_{s h}^{*}, t_{s h}, t_{s h+1}\right) .
\end{aligned}
$$

In the previous algorithm, $M^{i}$ corresponds to the QuasiNewtonian procedure proposed by Broyden-FletcherGoldberg-Shamo (BFGS) for updating the approximation of the inverse of the Hessian of $J($.$) [8], in the$ minimization of the criterion (6). The convergence of the estimations carried out with MHSE is directly related to the convergence of the optimization algorithm used to solve (5) (for details see [9]).

\section{MATHEMATICAL MODEL OF THE INDUCTION MOTOR}

The mathematical model of the IM that we will use is also described in [1], [7], [10]. In this model the voltage equations can be written in a stationary reference frame $\alpha \beta 0$. This can be done by using the following coordinate transformation:

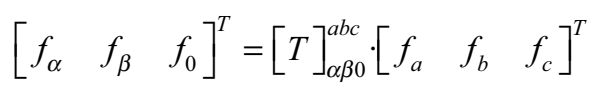

In this equation the matrix $[T]_{\alpha \beta 0}^{a b c}$ can be described by:

$$
[T]_{\alpha \beta 0}^{a b c}=\sqrt{\frac{2}{3}} \cdot\left[\begin{array}{ccc}
1 & -\frac{1}{2} & -\frac{1}{2} \\
0 & \frac{\sqrt{3}}{2} & -\frac{\sqrt{3}}{2} \\
\frac{1}{\sqrt{6}} & \frac{1}{\sqrt{6}} & \frac{1}{\sqrt{6}}
\end{array}\right]
$$

Note that the subzero variable is equal to zero due to the balancing conditions. In (8) $f$ can represent voltages, currents or fluxes, $(v, i, \phi)$ respectively. From this it is possible to obtain the following voltage equations for the rotor and stator [7]:

$$
\rho \bar{\phi}_{s \alpha \beta}=\bar{v}_{s \alpha \beta}-R_{s} \bar{i}_{s \alpha \beta}
$$

$$
\rho \bar{\phi}_{r \alpha \beta}=-\left(T_{r}-j w_{r}\right) \bar{\phi}_{r \alpha \beta}+T_{r} L_{m} \bar{i}_{s \alpha \beta}
$$

In (10) and (11), $\rho$ represents the operator $d / d t$. $L_{s}$, $L_{r}, L_{m}$ are the inductances of the stator, the rotor and the magnetization respectively. $R_{s}, R_{r}$ represent the stator and rotor resistances respectively. Finally, the equations $T_{r}=R_{r} / L_{r}$ and $\sigma=1-L_{m}^{2} / L_{s} L_{r}$ can be verified, where $w_{r}$ represents the angular velocity of the rotor. Considering these equivalencies, the mechanical motion of the system is described by:

$$
\rho w_{r}=\frac{n_{p}\left(T_{e}-T_{L}\right)}{I}-\frac{R_{w} w_{r}}{I}
$$

Considering the previous model in a rotational reference frame $d-q$, oriented in such a manner that the flux vector $\bar{\phi}_{r \alpha \beta}$ of the rotor has axis direction $d$, an additional simplification of the mathematical model can be made. This simplification shows the independence between the angle of the magnetic axis of the rotor and the magnetic axis of the stator. The transformation of the coordinates from the variables $\alpha \beta$ to the reference frame $d-q$ is given by the following form:

$$
\left[\begin{array}{ll}
f_{d} & f_{q}
\end{array}\right]^{T}=\left[\begin{array}{l}
T \\
]_{d q}^{\alpha \beta}
\end{array}\left[\begin{array}{ll}
f_{\alpha} & f_{\beta}
\end{array}\right]^{T}\right.
$$

In (13), the matrix $[T]_{d q}^{\alpha \beta}$ can be written as: 
Taking these considerations into account, a mathematical model of the IM can be obtained [1], [13], which can be expressed as follows:

$$
\dot{x}=f(x)+\tilde{g} \cdot u
$$

In which (15) is completely determined by the following relationships where $i_{s d}, i_{s q}$ represent the stator currents, $\phi_{r d}, \phi_{r q}$ the rotor fluxes, $u_{s d}, u_{s q}$ the stator voltages, $I$ the moment of inertia of the machine, $n_{p}$ the number of pole pairs, $T_{L}$ the load torque, $T_{r}$ the rotor time constant and $R_{w}$ the friction associated with motion.

$$
\begin{aligned}
& x=\left[\begin{array}{lllll}
i_{s d} & i_{s q} & \phi_{r d} & \phi_{r q} & w_{r}
\end{array}\right]^{T} \\
& u=\left[\begin{array}{ll}
u_{s d} & u_{s q}
\end{array}\right]^{T} \\
& f(x)=\left[\begin{array}{c}
-\gamma i_{s d}+\frac{K}{T_{r}} \phi_{r d}+\rho K w_{r} \phi_{r q} \\
-\gamma i_{s q}-\rho K w_{r} \phi_{r d}+\frac{K}{T_{r}} \phi_{r q} \\
\frac{L_{m}}{T_{r}} i_{s d}-\frac{1}{T_{r}} \phi_{r d}-\rho w_{r} \phi_{r q} \\
\frac{L_{m}}{T_{r}} i_{s q}+\rho w_{r} \phi_{r d}-\frac{1}{T_{r}} \phi_{r q} \\
n_{p} \frac{L_{m}}{I \cdot L_{r}}\left(\phi_{r d} i_{s q}-\phi_{r q} i_{s d}\right)-\frac{1}{I}\left(T_{L}+R_{w} w_{r}\right)
\end{array}\right] \\
& \tilde{g}=\left[\begin{array}{ccccc}
\frac{1}{\sigma L_{s}} & 0 & 0 & 0 & 0 \\
0 & \frac{1}{\sigma L_{s}} & 0 & 0 & 0
\end{array}\right]^{T} \\
& K=\frac{L_{m}}{\sigma L_{s} L_{r}} \quad \gamma=\frac{R_{s}}{\sigma L_{s}}+\frac{R_{r} L_{m}^{2}}{\sigma L_{s} L_{r}^{2}}
\end{aligned}
$$

Remark: In $f(x)$, the load torque $T_{L}$ is employed as a known parameter in order to estimate IM states using the MHSE method. This strategy has been used by several different authors; see for example [1], [2], [11], [13].

\section{SIMULATION}

The application of the MHSE method to an IM has been carried out through a computer simulation. The IM model considered for this purpose is equivalent to a $1.1 \mathrm{KW}$, $220 / 380 \mathrm{~V}, 50 \mathrm{~Hz}$ and $1500 \mathrm{rpm}$ motor. Additionally this model has the following typical characteristics:

$$
\begin{array}{ll}
R_{r}=3.6 \Omega & J=0.015 \mathrm{Kgm}^{2} \\
R_{s}=8 \Omega & R_{w}=0.005 \mathrm{Nm} \\
L_{r}=0.47 \mathrm{H} & n_{p}=2 \\
L_{s}=0.47 \mathrm{H} & L_{m}=0.452 \mathrm{H}
\end{array}
$$

For the estimation trials of the angular velocity $w$ of the IM rotor, the following three possibilities have been considered:

a) No perturbations.

b) Instantaneous perturbations of the output $w$ of the simulated system.

c) Cyclic perturbations of the output $w$ of the simulated system.

Figure 1 corresponds to case a) (no perturbations) and shows excellent coherence between the simulated angular velocity $w$ of the IM rotor, and its corresponding estimation by the proposed MHSE method. The convergence time for the estimation method is less than $t=0.2$ seconds.

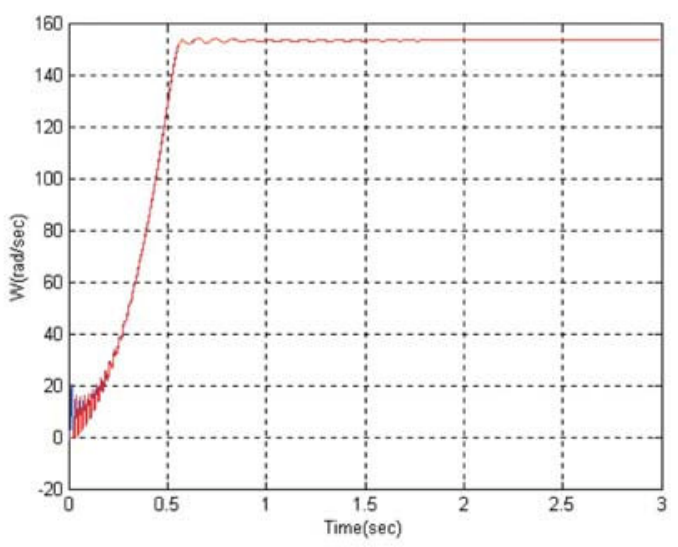

Fig. 1 Angular velocity for the IM under consideration -reference (in blue), and estimated (in red).

If we consider more realistic working conditions for an $\mathrm{IM}$, as for example case b), figure 2 presents the estimates achieved for four consecutive torque perturbations. The first three perturbations, typical of industrial processes, occur for $t=0.8, t=1.2$ and $t=1.8$ seconds respectively in the simulation. The fourth considers an instantaneous perturbation in velocity (representing a 10\% loss of velocity) at $t=2.5$ seconds. For each of these 
perturbations, the coherence obtained between the estimation and the simulated process, and the convergence times are excellent.

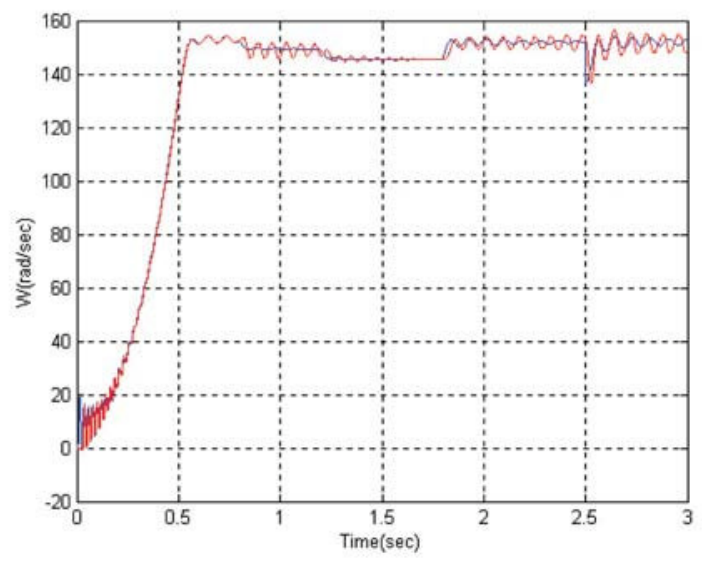

Fig. 2 Estimation considering perturbations in $w$ : Reference (in blue) and estimation (in red)

Finally figure 3 also shows good, coherent results with good convergence times between the obtained estimation and the theoretical simulation of the process for each of the perturbations. This process considers cyclical variations in torque as perturbations, with $t=0.9$ seconds, such as the start up of a compressor.

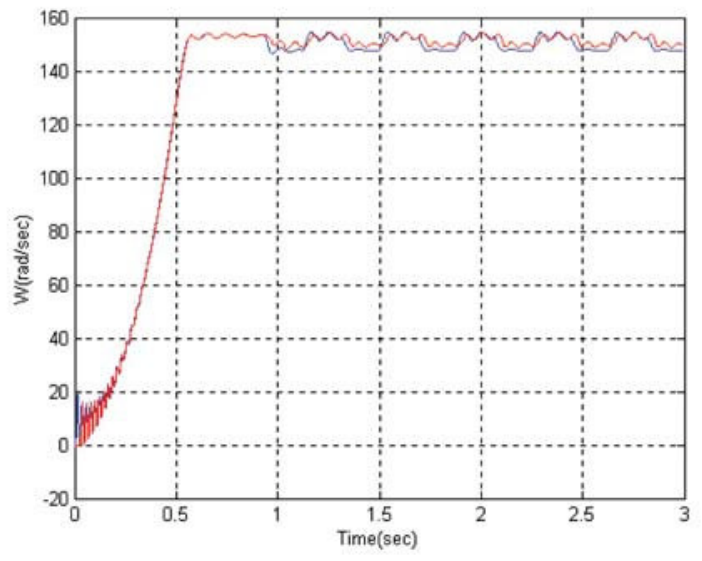

Fig. 3 Simulation (in blue) and estimation $w$ (in red) for cyclical variations in torque

\section{CONCLUSIONS}

This paper presents an innovative method for the estimation of states in an IM based on non-linear optimization. This method is called MHSE, and is based on the minimization of a non-linear criterion. In this case, the classic Quasi-Newtonian BFGS method has been used to solve the proposed optimization problem. This problem derives from the need to carry out estimations of the angular velocity of the rotor in an IM. The simulations of three possible cases have been presented, and all show an excellent coherence between simulated and estimated process variables, as well as a good response time. These features lead us to believe that an experimental application of the MHSE technique, together with the control laws, may produce industrially useful results.

\section{FUTURE WORK}

It is necessary to compare the results obtained and those for other non-linear estimation methods, such as EKF (Extended Kalman Filter) and UKF (Unscented Kalman Filter), especially with respect to response times and precision.

\section{ACKNOWLEDGEMENTS}

The authors wish to thank the Department of Electrical Engineering at the Universidad de Santiago de Chile, the Fondecyt Project 1030067, and the Computer and Information Engineering Department at the Universidad de Tarapacá for their support which enabled this research project to take place. The second author also wishes to thank the "Fundación Andes" for the grant C-14060/17 "Inicio de carrera para jovenes investigadores" which also partially supported this work.

\section{REFERENCES}

[1] M. K. Maaziz, E. Mendes, P. Boucher. "A new nonlinear multivariable control strategy of induction motors". Control Engineering Practice. Vol. 10, pp. 605-613. 2002.

[2] K.L. Shi, T.F. Chan, Y.K. Wong, S.L. Ho. "Speed estimation of an induction motor drive using an optimized extended Kalman filter". IEEE Transactions on Industrial Electronics. Vol.49, Iss.1, pp.124-133, Feb. 2002.

[3] B. Akin, U. Orguner, A. Ersak. "A comparative study on Kalman filtering techniques designed for state estimation of industrial AC drive systems". Proceedings of the IEEE International Conference on Mechatronics. ICM '04. June 35, pp. 439- 445. 2004. 
[4] D. Aeyels. "On the number of samples necessary to achieve observability". Syst. Contr. Lett. Vol. 1, pp. 92-94. 1981.

[5] C. Manes, F. Parasiliti, and M. Tursini. "A comparative study of rotor flux estimation in induction motors with a nonlinear observer and the extended Kalman filter". in Proc. IEEE IECON'94, pp. 2149-2154. 1994.

[6] S. Chávez-Velázquez, R. Alejos-Palomares, A. Nava-Segura. "Speed Estimation for an Induction Motor Using the Extended Kalman Filter". Proceedings of the 14th International Conference on Electronics, Communications and Computers (CONIELECOMP'04). IEEE Computer Society. 2004.

[7] L. Salvatore, S. Stasi, and L. Tarchioni. "A new EKF-based algorithm for flux estimation in induction machines". IEEE Trans. Ind. Electron., Vol. 40, pp. 496-504. Oct. 1993.

[8] D. Luenberger "Linear and Nonlinear Programming”. Second edition. Addison-Wesley Publishing Company, Inc. 1984.

[9] L. Boillereaux. "Estimation d'état des procèdes non-linéaires: Méthode a horizon glissant avec indicateur de qualité". PhD thesis, Laboratory of Automatic Control of Grenoble (LAG). Francia. 1996.

[10] I. Barbi. "Teoria Fundamental do Motor de Indução". Florianopolis: Ed. da UFSC, Electrobrás. 1985.

[11] M. Alamir. "Sensitivity analysis in simultaneous state/parameter estimation for induction motors". International Journal of Control. Vol 75. $\mathrm{N}^{\mathrm{o}} 10$, pp. 753-758. 2002.

[12] M. Alamir. "Optimization Based Nonlinear Observers Revisited". International Journal of Control 72. No 13, 1204-1217. 1999.

[13] P. Cataldi, W. Geri, M. Montanari and A. Tilli, "A new adaptive approach for on-line parameter and state estimation of induction motors". Control Engineering Practice. Vol. 13, pp. 81-94. 2005.

[14] G. Valdés-González. “Comparación de algoritmos no lineales de estimación de estados aplicados a un motor de inducción: estudio basado en simulación computacional". MSc thesis (in Electrical Engineering). Electrical Department. Universidad de Santiago de Chile. 2005. 